\title{
On the Tuning of Predictive Controllers: Application of Generalized Benders Decomposition to the ELOC Problem
}

\author{
Jin Zhang, Benjamin P. Omell, Donald J. Chmielewski* \\ Department of Chemical $\&$ Biological Engineering, Illinois Institute of Technology, Chicago, IL 60616
}

\begin{abstract}
This work investigates the computational procedures used to obtain global solution to the Economic Linear Optimal Control (ELOC) problem. The proposed method employs the Generalized Benders Decomposition (GBD) algorithm. Compared to the previous branch and bound approach, a naive application of GBD to the ELOC problem will improve computational performance, due to less frequent calls to computationally slow Semi-Definite Programming (SDP) routines. However, the reverse-convex constraints of the original problem will reappear in the relaxed master problem. In response, a convexification of the relaxed master constraints has been developed and proven to preserve global solution characteristics. The result is a multi-fold improvement in computational performance. A technological benefit of decomposing the problem into steady-state and dynamic parts is the ability to utilize nonlinear steady-state models, since the relaxed master problem is free of SDP type constraints and can be solved using any global nonlinear programming algorithm.

Keywords: Stochastic Control; Optimization; Generalized Benders Decomposition; Linear Matrix Inequalities
\end{abstract}

\section{Introduction}

In the first instalment, [1], of this series on the tuning of predictive controllers, [1-3], it was shown that the linear feedback generated by the Minimum Variance Covariance Constrained

*Corresponding author. phone: 312-567-3537, fax: 312-567-8874, email: chmielewski@iit.edu 
Control $\left(M V C^{3}\right)$ problem is guaranteed to be in the family of Linear Quadratic Regulator (LQR) feedback policies. In addition, using the inverse optimality results of [1], one could generate quadratic objective function weights such that if used within the LQR problem, the resulting controller would be identical to the $M V C^{3}$ policy. It was then postulated that such a scheme could be used within a predictive controller context to tune the controller (i.e., select quadratic objective function weights) such that the weights and the point-wise-in-time constraints would be in alignment.

In the second instalment of the series, [2], the $M V C^{3}$ problem was recast into the framework of Backed-off Operating Point (BOP) selection, [4-11]. In contrast to the BOP selection procedure of [7], which assumed a pre-specified linear feedback policy, the Minimally Backed-off Operating Point (MBOP) selection procedure of [2] allowed for the linear feedback to be selected simultaneous to the BOP. The term minimal was appended to BOP to indicate that the feasible set of BOPs was expanded by including all feasible linear feedback policies. The precise definition of a feasible linear feedback will be given in the next sub-section.

An important aspect of casting the $M V C^{3}$ problem into the BOP selection framework is that the linear feedback resulting from the MBOP problem will be engendered with the economic motives of the BOP problem. In essence, the MBOP generated linear feedback is aligned with the economic objective function of the BOP problem. Thus, a more descriptive name for the MBOP problem is the Economic Linear Optimal Control (ELOC) problem, which will generate a linear feedback policy termed the ELOC policy. Given the ELOC policy, one can again use the inverse optimality results of [1] to generate a predictive form of the ELOC policy. If one then imposes point-wise-in-time constraints to the (unconstrained) predictive form of the ELOC, the resulting controller is denoted as the Constrained ELOC policy. In this case the objective function weights of the predictive controller are tuned to be aligned, not only with the constraints, but also aligned with the economics of the process.

In the third instalment, [3], the practical aspects of implementing the Constrained ELOC policy on a chemical process are illustrated. In particular, the issues of disturbance modeling, 
measurement feedback, soft constraints, and computational delay are all elucidated. Use of ELOC and Constrained ELOC within a variety of other applications can be found in [12-19].

As indicated in [2], the ELOC problem possesses a set of reverse-convex constraints. To overcome this non-convexity, [2] advocates use of a branch-and-bound search procedure to obtain a global solution. Alternative methods for solving the ELOC problem can be found in $[20,21]$. A particular drawback of the branch-and-bound approach (other than the slow convergence rate) is that the steady-state process model (used within the BOP aspect of the ELOC) must either be linear or be such that it can be converted to an appropriate convex equivalent, see for example $[14,15]$. This restriction is more a of a technological issue and stems from the fact that characterization of feasible feedback policies manifests as a set of Linear Matrix Inequality (LMI) constraints that require a Semi-Definite Programming (SDP) solver for enforcement. Unfortunately, all currently available SDP solvers are unable to enforce general nonlinear constraints, which leads to the above limitation with respect to steady-state process models. The proposed Generalized Benders Decomposition (GBD) approach to solving the ELOC problem will remove this technological limitation while providing significant improvements in solution time.

In the remainder of this section, the method of [2] will be reviewed and the illustrative example of a Mass Spring Damper system will be introduced. Then, the GBD algorithm will be reviewed. In section 2, the GBD algorithm will be applied to the ELOC problem. The result will be the creation of a relaxed master problem that does not possess any LMI constraints and thus does not require the use of a Semi-Definite Programming (SDP) algorithm. However, this relaxed master problem will also be shown to possess reverseconvex constraints and thus will require application of the branch and bound algorithm. In section 3, a method to convexify the relaxed master constraints will be proposed and proven to preserve the global solution characteristics of the GBD algorithm. Section 4 will illustrate the computational scalability of the proposed method using a reaction process as well as a manufacturing system. In addition, section 4 will illustrate the ability of the new algorithm 
to employ nonlinear steady-state process models.

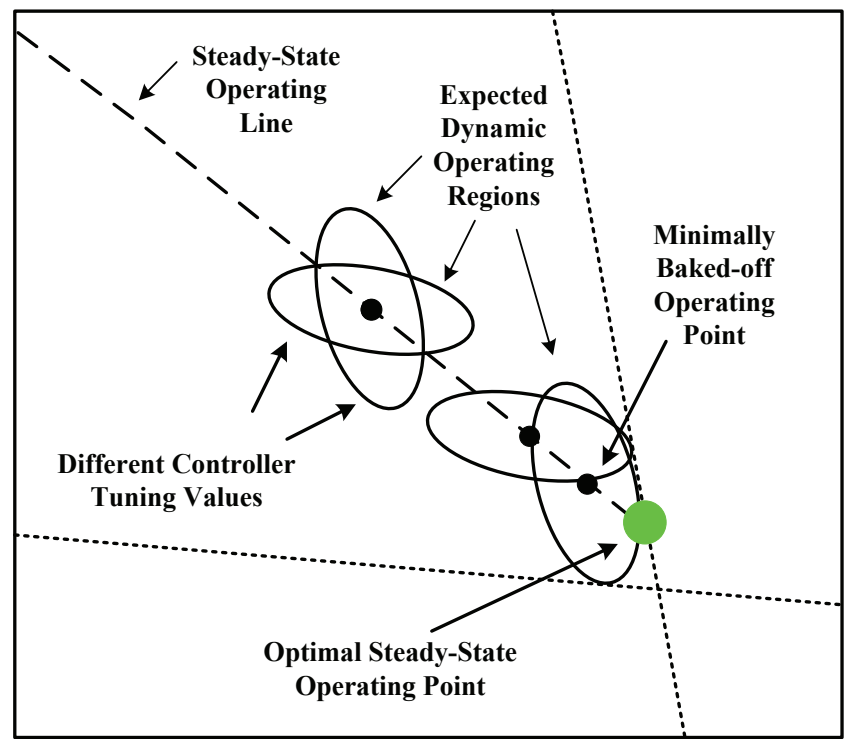

Figure 1: Illustration of the MBOP/ELOC problem.

\subsection{Review of the ELOC Problem}

Consider a nonlinear process model: $\dot{s}=f(s, m, p), q=h(s, m, p)$, and $q^{\text {min }} \leq q \leq q^{\max }$ where $s, m, p$ and $q$ are the state, manipulated, disturbance and performance variables (each a column vector of dimension $n_{s}, n_{m}, n_{p}$ and $n_{q}$, respectively). The Optimal Steady State Operating Point (OSSOP) can be found by the following nonlinear optimization problem:

$$
\min _{\substack{s^{s s}, m^{s s} \\ q^{\min } \leq q^{s s} \leq q^{\max }}}\left\{g\left(q^{s s}\right)\right\} \quad \text { s.t. } 0=f\left(s^{s s}, m^{s s}, \bar{p}\right) \quad q=h\left(s^{s s}, m^{s s}, \bar{p}\right)
$$

where $g(\cdot)$ is the operating cost function and $\bar{p}$ is the steady-state or nominal value of $p$. The solution to this nonlinear optimization is denoted by the triple $\left(s^{O S S O P}, m^{O S S O P}, q^{O S S O P}\right)$. Unfortunately, operation of the process at the OSSOP is expected to result in significant constraint violations during dynamic operation (i.e., $q(t)$ will not satisfy $q^{\min } \leq q \leq q^{\max }$ ). This is due to the fact that non-steady-state disturbances (i.e., $p \neq \bar{p}$ ) will cause $q$ to deviate from $q^{O S S O P}$. In fact, during dynamic operation the output $q$ will reside in a region 
surrounding $q^{\text {OSSOP }}$. This region is denoted as the Expected Dynamic Operation Region (EDOR) and is illustrated in Figure 1. To reduce the occurrence of constraint violations the actual steady-state operating point of the controller will be selected to be a Backed-off Operating Point (BOP) that is some distance from the operating constraints, but still close to the OSSOP. The BOP is denoted as $\left(s^{B O P}, m^{B O P}, q^{B O P}\right)$, and must satisfy the steady-state relations, $0=f\left(s^{B O P}, m^{B O P}, \bar{p}\right), q^{B O P}=h\left(s^{B O P}, m^{B O P}, \bar{p}\right)$ and $q^{\text {min }} \leq q^{B O P} \leq q^{\max }$.

Turning to the dynamic perspective, the BOP will serve as the target point for the controller in that it will seek to drive the system toward the BOP. Thus, the variables seen by the controller should be deviation variables with respect to the BOP, defined as $\tilde{s}=s-s^{B O P}, \tilde{m}=m-m^{B O P}, \tilde{p}=p-p^{B O P}$, and $\tilde{q}=q-q^{B O P}$. Then, linearization of the original nonlinear dynamic model results in $\dot{\tilde{s}}=A \tilde{s}+B \tilde{m}+G \tilde{p}, \tilde{q}=D_{s} \tilde{s}+D_{m} \tilde{m}+D_{p} \tilde{p}$ where $A, B, G, D_{s}, D_{m}$ and $D_{p}$, are the system matrices of the process linearized around the OSSOP. Now assume a controller of the form $\tilde{m}=L \tilde{s}$, where $L$ is to be determined. Furthermore, assume $\tilde{p}$ is a zero-mean white noise process with a spectral density $S_{p}$. Then, the EDOR can be characterized by the following relations:

$$
\begin{aligned}
& \Sigma_{s}(A+B L)^{T}+(A+B L) \Sigma_{s}+G S_{p} G^{T}=0 \\
& \Sigma_{q}=\left(D_{s}+D_{m} L\right) \Sigma_{s}\left(D_{s}+D_{m} L\right)^{T}+D_{p} S_{p} D_{p}^{T} \\
& \zeta_{j}=\rho_{j} \Sigma_{q} \rho_{j}^{T}, j=1 \ldots n_{q}
\end{aligned}
$$

where $\Sigma_{s}$ and $\Sigma_{q}$ are the covariance matrices associated with $s$ and $q$ and $\rho_{j}$ is the $j^{\text {th }}$ row of an $n_{q} \times n_{q}$ identity matrix. Specifically, the size of the EDOR in the $j^{\text {th }}$ direction is defined as $\sqrt{\zeta_{j}}$. If one were to impose upper bounds to the size of the EDOR in each direction (i.e., $\sqrt{\zeta_{j}} \leq \bar{\sigma}_{j}$ ), then there will be a limited set of controllers, $L$, capable of generating an EDOR that satisfies these constraints. This set of covariance constrained feasible controllers is central to the $M V C^{3}$ problem and ultimately to the generation of an ELOC policy. Theorem 3.1 of [1] indicates that the nonlinear equalities of (2), (3), (4) hold 
if and only if the convex Linear Matrix Inequalities (LMI) of (9) and (10) are satisfied.

The ELOC problem is then a result of combining the dynamic perspective (EDOR) with the steady-state perspective (BOP), by excluding any BOP such that the associated EDOR is not contained in the region defined by $q^{\min }$ and $q^{\max }$. Specifically, the following must be enforced: $\sqrt{\zeta_{j}} \leq q_{j}^{B O P}-q_{j}^{\min }$ and $\sqrt{\zeta_{j}} \leq q_{j}^{\max }-q_{j}^{B O P}, j=1 \ldots n_{q}$.

In sum, the ELOC problem is defined as

$$
\left.\begin{array}{l}
\min _{\substack{s^{B O P}, m^{B O P}, q^{B O P}, \sigma>0, \zeta>0, M_{0}>0, M_{1}}}\left\{g\left(q^{B O P}\right)\right\} \text { s.t. } \\
0=f\left(s^{B O P}, m^{B O P}, \bar{p}\right), q^{B O P}=h\left(s^{B O P}, m^{B O P}, \bar{p}\right) \\
\sigma \leq q^{\max }-q^{B O P}, \sigma \leq q^{B O P}-q^{\min } \\
\zeta \leq \sigma^{2} \\
\left(A M_{0}+B M_{1}\right)+\left(A M_{0}+B M_{1}\right)^{T}+G S_{p} G^{T}<0 \\
{\left[\begin{array}{cc}
\zeta_{j}-\rho_{j} D_{p} S_{p} D_{p}^{T} \rho_{j}^{T} & \rho_{j}\left(D_{s} M_{0}+D_{m} M_{1}\right)
\end{array}\right]>0, j=1 \ldots n_{q}} \\
\left(D_{s} M_{0}+D_{m} M_{1}\right)^{T} \rho_{j}^{T}
\end{array}\right]
$$

\section{Remarks:}

(1) Dummy variables $\sigma_{j}$ are introduced to the formulation and are restricted to be such that (7) and (8) hold. These can be interpreted as the standard deviation of output $j$, but are actually upper bounds to the standard deviation, since (8) is an inequality. These variables will be central to the subsequent GBD approach.

(2) Note the abuse of notation in that $\sigma^{2}$ is used to indicate $\left[\begin{array}{lll}\sigma_{1}^{2} & \sigma_{2}^{2} \ldots \sigma_{n_{q}}^{2}\end{array}\right]^{T}$. In section 3, the notation $\sqrt{\zeta}=\left[\begin{array}{lll}\sqrt{\zeta_{1}} & \sqrt{\zeta_{2}} \ldots \sqrt{\zeta_{n_{q}}}\end{array}\right]^{T}$ will also be used.

(3) If $M_{0}^{*}$ and $M_{1}^{*}$ are from the solution to Problem (5), then the ELOC policy is calculated as $L_{E L O C}=M_{1}^{*}\left(M_{0}^{*}\right)^{-1}$.

(4) In the original formulation of [2], constraint (11) was not included. While this constraint is redundant with (7) and (8), it will be of utility in the subsequent developments. 
Example 1a: Consider the following Mass-Spring-Damper process

$$
\begin{gathered}
g(q)=-q_{1}, \quad f(s, m, p)=\left[\begin{array}{c}
s_{2} \\
-3 s_{1}-2 s_{2}+m+p
\end{array}\right], \bar{p}=-9.8 \\
h(s, m, p)=\left[\begin{array}{c}
s_{1} \\
m
\end{array}\right], \quad q^{\min }=\left[\begin{array}{c}
-1 \\
0
\end{array}\right], q^{\max }=\left[\begin{array}{c}
1 \\
15
\end{array}\right]
\end{gathered}
$$

The OSSOP is found to be $s_{1}^{O S S O P}=1, s_{2}^{O S S O P}=0$, and $m^{O S S O P}=12.8$. We can now convert the dynamic process model to deviation variables, arriving at the following system matrices

$$
A=\left[\begin{array}{cc}
0 & 1 \\
-3 & 2
\end{array}\right], B=\left[\begin{array}{l}
0 \\
1
\end{array}\right], G=\left[\begin{array}{l}
0 \\
1
\end{array}\right], D_{x}=\left[\begin{array}{ll}
1 & 0 \\
0 & 0
\end{array}\right], D_{u}=\left[\begin{array}{l}
0 \\
1
\end{array}\right]
$$

If the spectral density of the disturbance is $S_{p}=10$, then the BOP portion of the ELOC solution is $s_{1}^{B O P}=0.64, s_{2}^{B O P}=0$, and $m^{B O P}=11.7$. Furthermore, if $M_{0}^{*}$ and $M_{1}^{*}$ are from the ELOC problem solution then the ELOC policy is $L_{E L O C}=M_{1}^{*}\left(M_{0}^{*}\right)^{-1}=-\left[\begin{array}{ll}6.43 & 2.11\end{array}\right]$.

This solution is arrived at via the branch and bound procedure discussed in [2]. A key element of the example is that $f(s, m, \bar{p}), h(s, m, \bar{p})$ and $g(q)$ are linear functions of $s, m$, and $q$. Since the LMI constraints (9) and (10) are convex, only the reverse-convex constraints of (8) present a computational challenge. In the procedure of [2] the $\sigma_{j}$ 's are designated as branching variables. Then, during the investigation of the nodes the inequalities of (8) are replaced with $\zeta_{j} \leq m_{j} \sigma_{j}+b_{j}$, where $m_{j}$ and $b_{j}$ are parameters within the sub-problem optimizations. The global solution to this simple problem is depicted in Figure 2 (left). The algorithm required 7 branch and bound iterations and a solution time of 12 seconds. Notice that the elliptical EDOR is completely contained within the constraints, (dashed line) and the BOP (center of the ellipse) is as close as possible to the OSSOP. To provide a bit of 
insight into the branch and bound procedure (as well as the subsequent discussion) consider the impact of the under- and over-estimate approximations. In the under-estimate case, the ellipse is allowed to extend beyond the bounds slightly, and thus the BOP is allowed to be slightly closer to the OSSOP. In the over-estimate case, the ellipse must be a small distance away from the bounds and thus a bit further from the OSSOP.

Let us also take this opportunity to illustrate an important set that will be central to the subsequent discussion. This is the set of $\sigma$ 's such that there exists a feasible EDOR, $V=\left\{\sigma \geq 0 \mid \exists \zeta, M_{0}\right.$, and $M_{1}$, satisfying (8),(9),(10) and (11) $\}$. For this simple two dimensional example the boundary of this set can be calculated from $B_{v}\left(\bar{\sigma}_{1}\right)=\min \left\{\sigma_{2} \mid \sigma_{1} \leq\right.$ $\bar{\sigma}_{1}$ and (8),(9),(10) and (11)\} and is depicted in Figure 2 (right), along with the ELOC problem solution.
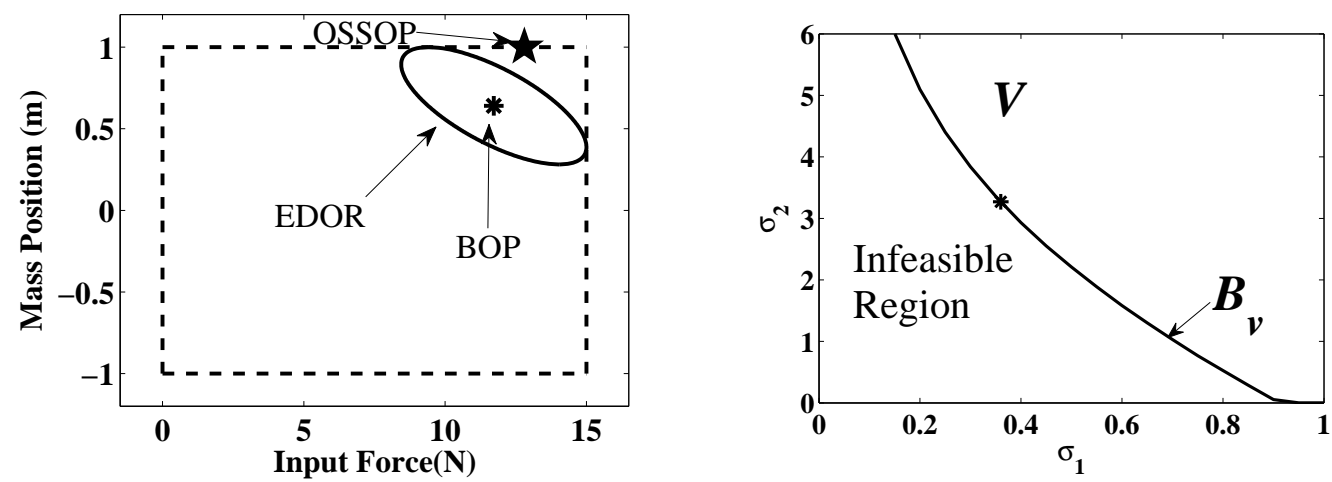

Figure 2: The ELOC solution (left) and the set $V$ (right), both for Example 1a.

\subsection{Review of Generalized Bender's Decomposition}

Consider the following optimization problem

$$
\min _{x \in X, y \in Y} F(x, y) \text { s.t. } G(x, y) \leq 0
$$


Assume $y$ is a set of complicating variables in that for a given $\bar{y} \in V=\{y \mid G(x, y) \leq$ 0 for some $x \in X\}$, the following primal problem is easily solved:

$$
\min _{x \in X} F(x, \bar{y}) \quad \text { s.t. } \quad G(x, \bar{y}) \leq 0
$$

If $X$ is a convex set and both $G(x, y)$ and $F(x, y)$ are convex in $x$, then problem (12) is equivalent to the following master problem (see [22] for details):

$$
\min _{y_{0}, y \in Y}\left\{y_{0} \text { s.t. } L^{*}(y, u) \leq y_{0}, L_{*}(y, \lambda) \leq 0 \text { for all } u \geq 0 \text { and } \lambda \in \Lambda\right\}
$$

where

$$
\begin{aligned}
L^{*}(y, u) & =\min _{x \in X}\left\{F(x, y)+u^{T} G(x, y)\right\} \\
L_{*}(y, \lambda) & =\min _{x \in X}\left\{\lambda^{T} G(x, y)\right\} \\
\Lambda & =\left\{\lambda \geq 0 \mid 1-\lambda^{T} \underline{1}=0\right\}, \quad \underline{1}=\left[\begin{array}{lll}
1 & 1 & \ldots
\end{array}\right]^{T}
\end{aligned}
$$

The GBD algorithm is then based on the following relaxed master problem

$$
\begin{aligned}
& \min _{y_{0}, y \in Y} y_{0} \\
& L^{*}\left(y, u^{\left(k_{1}\right)}\right) \leq y_{0} \quad k_{1}=1 \ldots K_{1} \\
& L_{*}\left(y, \lambda^{\left(k_{2}\right)}\right) \leq 0 \quad k_{2}=1 \ldots K_{2}
\end{aligned}
$$

where $u^{\left(k_{1}\right)}$ and $\lambda^{\left(k_{2}\right)}$ are selected by the following procedure:

1. Initialize by selecting a point $\bar{y} \in Y \cap V$. Obtain the solution to the primal problem, $x^{*}$, along with its multipliers, $u^{*}$. Set $K_{1}=1, K_{2}=0, u^{(1)}=u^{*}, U B D=F\left(x^{*}, \bar{y}\right)$ and determine $L^{*}\left(y, u^{(1)}\right)$.

2. Obtain the global solution to the relaxed master problem $\left(y_{0}^{*}, y^{*}\right)$. Set $L B D=y_{0}^{*}$. If $U B D \leq L B D+\epsilon$, the problem has converged. 
3. Set $\bar{y}=y^{*}$. If the primal is feasible go to step 3a; if infeasible go to step 3b.

3a. Obtain the solution to the primal problem along with its multipliers $\left(x^{*}, u^{*}\right)$. Set $K_{1}=K_{1}+1, u^{\left(K_{1}\right)}=u^{*}$ and determine $L^{*}\left(y, u^{\left(K_{1}\right)}\right)$. If $F\left(x^{*}, \bar{y}\right)<U B D$ set $U B D=F\left(x^{*}, \bar{y}\right)$. Return to step 2.

3b. Determine multipliers $\lambda \in \Lambda$ such that $\min _{x \in X}\left\{\lambda^{T} G(x, \bar{y})\right\}>0$. Set $K_{2}=K_{2}+1$, $\lambda^{\left(K_{2}\right)}=\lambda$ and determine $L_{*}\left(y, \lambda^{\left(K_{2}\right)}\right)$. Return to step 2.

Concerning step 3b, if $\bar{y} \notin V$ then there must exist $\lambda \in \Lambda$ s.t. $\min _{x \in X}\left\{\lambda^{T} G(x, \bar{y})\right\}>0$ (see [22] for details). Determining such a $\lambda$ will eliminate $\bar{y}$ from future searches over $y$ (and hopefully many other $y \notin V)$. To obtain such a $\lambda$ it has been suggested, [23, 24], to solve

$$
\begin{aligned}
& \max _{\lambda \geq 0}\left\{\min _{x \in X}\left\{\lambda^{T} G(x, \bar{y})\right\}\right\} \text { s.t. } 1-\lambda^{T} \underline{1}=0 \\
& =\min _{\alpha}\left\{\max _{\lambda \geq 0}\left\{\min _{x \in X}\left\{\lambda^{T} G(x, \bar{y})+\alpha\left(1-\lambda^{T} \underline{1}\right)\right\}\right\}\right\} \\
& =\min _{\alpha}\left\{\max _{\lambda \geq 0}\left\{\min _{x \in X}\left\{\alpha+\lambda^{T}(G(x, \bar{y})-\alpha \underline{1})\right\}\right\}\right\} \\
& =\min _{\alpha, x \in X}\{\alpha\} \text { s.t. } G(x, \bar{y})-\alpha \underline{1} \leq 0
\end{aligned}
$$

The first equality holds due to convexity of $\Lambda$, while the third equality holds due to the convexity of $G(x, y)$ with $x$. Then, since $X$ is a convex set, the solution to this problem is readily obtained and its associated multipliers compose the desired $\lambda$.

Concerning the computational feasibility of the algorithm it will be exceedingly helpful to be able to calculate $L^{*}$ and $L_{*}$ as explicit functions of $y$. One situation that guarantees satisfaction of this L-dual adequacy property is if the functions $F$ and $G$ are linearly separable: $F(x, y)=F_{1}(x)+F_{2}(y)$ and $G(x, y)=G_{1}(x)+G_{2}(y)$.

\section{Application of GBD to the ELOC Problem}

Looking back to Problem (5), it is observed that constraints (6) and (7) contain only the variables $s^{B O P}, m^{B O P}, q^{B O P}$ and $\sigma$, while constraints (9), (10) and (11) contain only 
the variables $\zeta, M_{0}$ and $M_{1}$. Constraint (8) connects the two sets by possessing both $\sigma$ and $\zeta$. Since constraints (9), (10) and (11) are convex, it is natural to assign $y^{T}=$

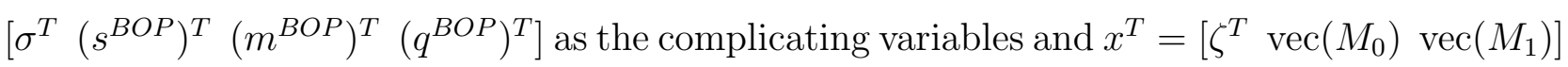
as the non-complicating variables. Thus, the ELOC problem can be stated as

$$
\min _{x \in X, y \in Y} F(x, y) \text { s.t. } G(x, y) \leq 0
$$

where $X=\{x \mid(9),(10)$ and (11) are satisfied $\}, Y=\{y \mid(6)$ and (7) are satisfied $\}$, $F(x, y)=g\left(q^{B O P}\right)$, and $G(x, y)=\zeta-\sigma^{2}$. Clearly, $F(x, y), G(x, y)$ and $X$ are convex with respect to $x$ and thus no duality gap will exist. In addition, $F(x, y)$ and $G(x, y)$ are linearly separable which will provide L-dual-adequacy to the algorithm.

Using these definitions the primal problem is

$$
\min _{x \in X} g\left(\bar{q}^{B O P}\right) \text { s.t. } \zeta-\bar{\sigma}^{2} \leq 0
$$

Since $x$ does not appear in the objective function, the primal is just a feasibility problem. As such, if the primal is feasible then the optimal multipliers, $u^{*}$, will be zero. If the primal is infeasible, then the following version of Problem (Q) should be solved.

$$
\min _{x \in X, \alpha}\{\alpha\} \quad \text { s.t. } \quad \zeta_{j}-\bar{\sigma}_{j}^{2}-\alpha \leq 0, \quad j=1 \ldots n_{q}
$$

Notice that this problem is convex in $x$ and $\alpha$, and thus can be solved to a global solution with relatively little effort.

Before considering the relaxed master problem, let us look at $L^{*}\left(y, u^{\left(k_{1}\right)}\right)$ and $L_{*}\left(y, \lambda^{\left(k_{2}\right)}\right)$. As stated above, if the primal is feasible, then the multipliers $u^{*}$ will be zero. Thus, for all $k_{1}$, $u^{\left(k_{1}\right)}=0$ and $L^{*}\left(y, u^{\left(k_{1}\right)}\right)=g\left(\bar{q}^{B O P}\right)$. To determine $L_{*}$ recall that each $\lambda^{\left(k_{2}\right)}$ is the solution to problem Q: $\max _{\lambda \in \Lambda} \min _{x \in X}\left\{\lambda^{T} G(x, \bar{y})\right\}=\max _{\lambda \in \Lambda}\left\{\min _{x \in X}\left\{\lambda^{T} \zeta\right\}-\lambda^{T} \bar{\sigma}^{2}\right\}$. Thus, if $\left(\zeta^{\left(k_{2}\right)}, \lambda^{\left(k_{2}\right)}\right)$ is the solution to problem $\mathrm{Q}$ then $L_{*}\left(y, \lambda^{\left(k_{2}\right)}\right)=\beta_{k_{2}}-\lambda^{\left(k_{2}\right) T} \sigma^{2}$ where $\beta_{k_{2}}=\lambda^{\left(k_{2}\right) T} \zeta^{\left(k_{2}\right)}$. Finally, 
the relaxed master problem is stated as

$$
\min _{y \in Y} g\left(q^{B O P}\right) \text { s.t. } L_{*}\left(y, \lambda^{\left(k_{2}\right)}\right)=\beta_{k_{2}}-\left(\lambda^{\left(k_{2}\right)}\right)^{T} \sigma^{2} \leq 0, \quad k_{2}=1 \ldots K_{2}
$$

Returning to the steps of the GBD algorithm, step 1 asks for a starting point $\bar{y} \in Y \cap V$, which indicates that $(\mathrm{P} 1)$ will be feasible and $u^{(1)}=0$. In addition, UBD is set equal to $g(\bar{q})$. Then, in step 2 one should solve RM1 with $K_{2}=0$. Clearly, this problem is identical to the OSSOP problem of (1). Thus, $\bar{y}$ is set equal to $y^{*}=\left[s^{O S S O P}, m^{O S S O P}, q^{O S S O P}, \sigma^{*}\right]$ where $\sigma^{*}$ is any feasible value for $\sigma$. It is noted that if any of the constraints $q_{j}^{\min } \leq q_{j}^{O S S O P} \leq q_{j}^{\max }$ are active, then $\sigma_{j}^{*}$ must be zero. Furthermore, LBD should be set equal to $g\left(q^{O S S O P}\right)$, the OSSOP operating cost. In the first execution of step 3 there are 2 options. If the primal is feasible, then UBD will be set equal to $g\left(q^{O S S O P}\right)$ and the algorithm will stop since $\mathrm{UBD}=\mathrm{LBD}$. If the primal is infeasible, then problem $(\mathrm{Q} 1)$ is solved to generate $\lambda^{(1)}$ and $\beta_{1}$. Assuming the pair $(A, B)$ is stabilizable, problem (Q1) should never be infeasible, unless constraint (11) cannot be satisfied. If (11) cannot be satisfied, then the overall ELOC problem is infeasible. Upon subsequent executions of step 2, there are 2 options; If RM1 is infeasible then the overall ELOC problem is infeasible. This will be true regardless of the iteration step and may occur several steps into the algorithm. If RM1 is feasible, then LBD will be updated (note that LBD is non-decreasing), and a new value for $\bar{y}$ will be sent to step 3.

\subsection{Calculation of Upper Bounds}

If at any point in the iterations the primal is feasible, then its solution will be equal to the LBD of the previous step 2. In this case, UBD will equal LBD and the algorithm should stop, as the optimal solution has been found. Unfortunately, this characteristic of UBD only being updated at the final step of the iteration will make it difficult to track the progress of the algorithm. As a substitute one could track the $\alpha^{*}$ solutions from problem (Q1). While this sequence is not guaranteed monotonic, it will give a qualitative indication of progress, as 
$\alpha^{*}$ will need to approach zero as the iterations approach the global solution. An alternative is to define an ad-hoc upper bound calculation. For example, using the $\zeta^{\left(k_{2}\right)}$ elements from the solution to problem (Q1) one can construct the following problem

$$
\min _{y \in Y} g\left(q^{B O P}\right) \text { s.t. } \sqrt{\zeta^{\left(k_{2}\right)}} \leq \sigma
$$

If this problem has a solution $\left(s^{*}, m^{*}, q^{*}, \sigma^{*}\right)$, then $g\left(q^{*}\right)$ will be an upper bound to the global solution, if $\zeta \hat{=}\left(\sigma^{*}\right)^{2} \in X$. This upper bound calculation procedure will be used in the subsequent examples.

As noted earlier, constraint (11) was not included in the formulation of [2]. Clearly, its addition to problem (5) will have no impact on the solution, since it is redundant with constraints (7) and (8). However, in Problem (Q1) constraint (7) is not present and a solution to Q1 could be found that violates (11) and causes problem (17) to immediately be infeasible. The addition of (11) to Q1 removes this possibility and tends to provide rather tight UBD values, as illustrated in the following examples.

Example 1b: Application of the GBD algorithm to the mass spring damper system generates the iteration result of Table 1. In this example the stopping criterion was set as $\epsilon=$ $0.01|\mathrm{LBD}|$. The plots of Figure 3 illustrate the results graphically. The top left plot indicates that LBD and UBD converge after 4 iterations. The top right plot depicts the first iteration solutions. The relaxed master has a solution $\left(\sigma_{1}^{*}, \sigma_{2}^{*}\right)=(0,0)$, the circle point. Problem Q1 has a solution $\zeta_{1}^{*}=\zeta_{2}^{*}=0.57$, which is depicted in the plot as the ${ }^{*}$ point, $\left(\sqrt{\zeta_{1}^{*}}, \sqrt{\zeta_{2}^{*}}\right)=(0.755,0.755)$. Then, using $\lambda^{(1)}$ and $\beta_{1}$ from Table $1, L_{*}\left(y, \lambda^{(1)}\right) \leq 0$ is

Table 1: GBD iteration results for Example 1b.

\begin{tabular}{cccccccc}
\hline \hline$k_{2}$ & $\mathrm{LBD}$ & $\mathrm{UBD}$ & $\lambda_{1}^{\left(k_{2}\right)}$ & $\lambda_{2}^{\left(k_{2}\right)}$ & $\left(\zeta_{1}^{*}\right)^{\frac{1}{2}}$ & $\left(\zeta_{2}^{*}\right)^{\frac{1}{2}}$ & $\beta_{k_{2}}$ \\
\hline 1 & -1.000 & -0.245 & 0.834 & 0.166 & 0.755 & 0.755 & 0.569 \\
2 & -0.733 & -0.466 & 0.959 & 0.041 & 0.534 & 1.987 & 0.435 \\
3 & -0.644 & -0.609 & 0.984 & 0.016 & 0.391 & 3.014 & 0.292 \\
4 & -0.641 & -0.640 & 0.988 & 0.012 & 0.360 & 3.269 & 0.261 \\
\hline
\end{tabular}



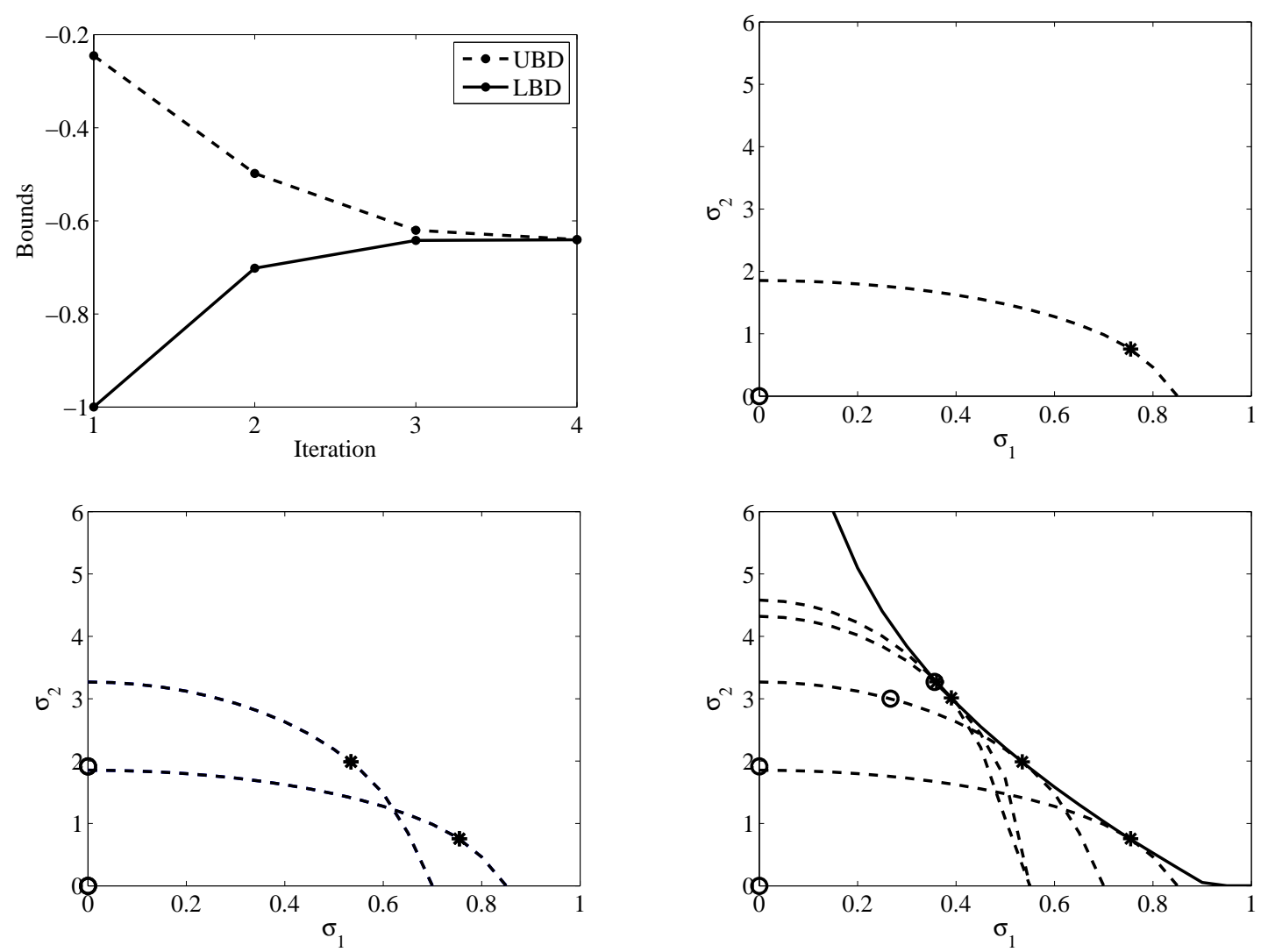

Figure 3: Illustration of GBD algorithm for Example 1b. Top-left: UBD and LBD vs. iteration number. Top-right: $L_{*}\left(y, \lambda^{(1)}\right) \leq 0$. Bottom-left: $L_{*}\left(y, \lambda^{(2)}\right) \leq 0$. Bottom-right: $L_{*}\left(y, \lambda^{\left(k_{2}\right)}\right) \leq 0$ for all iterations. The region above the solid line is the set $V$. The ${ }^{*}$ point represents the solution to problem (Q1), while the 'o' point represents the solution to (RM1).

displayed as the region above the dashed curve. The bottom left plot shows the solution of the second relaxed master iteration as the new circle point. The Q1 solution (the * point) and the $L_{*}\left(y, \lambda^{(2)}\right) \leq 0$ region are also shown. The bottom right plot depicts the results for all 4 iterations. This plot also shows the set $V$ as the region above the solid curve. This comparison clearly illustrates that each time a constraint $L_{*}\left(y, \lambda^{\left(k_{2}\right)}\right) \leq 0$ is added to the relaxed master, its feasible region becomes a better approximation of $V$. It also shows that the approximation need not be of high quality at points far from the solution point.

It is highlighted that the feasible region for each relaxed master problem is non-convex. This is clearly observed in the plots of Figure 3, but could also be noted by the nonconvexity of each $L_{*}\left(y, \lambda^{\left(k_{2}\right)}\right) \leq 0$ constraint in (RM1). Thus, to solve each of the relaxed 
master problems, a branch and bound algorithm had to be implemented to obtain its global solution. Each of these searches required roughly 20 iterations. The next section illustrates one approach to removal of this non-convexity in the relaxed master problem.

\section{Convexification of Relaxed Master Constraints}

Let us now consider the following restatement of the ELOC problem

$$
\min _{x \in X, y \in Y} F(x, y) \text { s.t. } G^{\prime}(x, y) \leq 0
$$

where $G^{\prime}(x, y)=\sqrt{\zeta}-\sigma$. Clearly, this problem is equivalent to problem (16), since for all $\zeta>0$ and $\sigma>0, \sqrt{\zeta} \leq \sigma$ if and only if $\zeta \leq \sigma^{2}$. Thus, since for all $\bar{\sigma}>0, X_{G^{\prime}}=$ $\left\{\zeta>0 \mid G^{\prime}(x, \bar{y}) \leq 0\right\}=\{\zeta>0 \mid G(x, \bar{y}) \leq 0\}$ is a convex region, there is no concern over a duality gap. The issue concerns the computational complexity of problem (Q) using $G^{\prime}(x, y)$ :

$$
\max _{\lambda \in \Lambda}\left\{\min _{x \in X}\left\{\sum_{j} \lambda_{j}\left(\sqrt{\zeta_{j}}-\bar{\sigma}_{j}\right)\right\}\right\}
$$

The following theorem will help alleviate this complexity.

Theorem 1 The solution to problem (Q2), $\left(\zeta_{j}^{*}, \lambda_{j}^{*}\right)$, is given by $\zeta_{j}^{*}=\zeta_{j}^{\prime *}$ and $\lambda_{j}^{*}=\lambda_{j}^{\prime *} \sqrt{\zeta_{j}^{\prime *}} / a$ where $a=\sum_{j} \lambda_{j}^{\prime} \sqrt{\zeta_{j}^{\prime *}}$ and $\left(\zeta_{j}^{\prime *}, \lambda_{j}^{\prime *}\right)$ is the solution to:

$$
\max _{\lambda^{\prime} \in \Lambda}\left\{\min _{x^{\prime} \in X}\left\{\sum_{j} \lambda_{j}^{\prime}\left(\zeta_{j}^{\prime}-\sqrt{\zeta_{j}^{\prime}} \bar{\sigma}_{j}\right)\right\}\right\}
$$

Proof. For any positive constant $a$, the solution to problem (Q2) is $\lambda^{*}=\hat{\lambda}^{*} / a$ where $\hat{\lambda}^{*}$ is 
the solution to

$$
\begin{aligned}
& \max _{\hat{\lambda} \geq 0}\left\{\min _{x \in X}\left\{\sum_{j} \hat{\lambda}_{j}\left(\sqrt{\zeta_{j}}-\bar{\sigma}_{j}\right)\right\} \text { s.t. } \sum_{j} \hat{\lambda}_{j}=a\right\} \\
= & \max _{\hat{\lambda} \geq 0}\left\{\min _{x \in X}\left\{\sum_{j} \frac{\hat{\lambda}_{j}}{\sqrt{\zeta_{j}}}\left(\zeta_{j}-\sqrt{\zeta_{j}} \bar{\sigma}_{j}\right)\right\} \text { s.t. } \sum_{j} \hat{\lambda}_{j}=a\right\} \\
= & \max _{\lambda^{\prime} \geq 0}\left\{\min _{x^{\prime} \in X}\left\{\sum_{j} \lambda_{j}^{\prime}\left(\zeta_{j}^{\prime}-\sqrt{\zeta_{j}^{\prime}} \bar{\sigma}_{j}\right)\right\} \text { s.t. } \sum_{j} \lambda_{j}^{\prime} \sqrt{\zeta_{j}^{\prime}}=a\right\}
\end{aligned}
$$

Since $a$ can be selected as any positive constant, the constraint $\sum_{j} \lambda_{j}^{\prime} \sqrt{\zeta_{j}^{\prime}}=a$ may be removed as long as $\sum_{j} \lambda_{j}^{\prime} \sqrt{\zeta_{j}^{\prime}}$ is guaranteed to be positive. Since $\zeta_{j}^{\prime}>0$ for all $j$, this guarantee is achieved by enforcing $\sum_{j} \lambda_{j}^{\prime}=1$. Thus, given the solution, $\left(\lambda^{\prime *}, \zeta^{\prime *}\right)$ of (Q3), the solution to (Q2) is $\zeta_{j}^{*}=\zeta_{j}^{* *}$ and $\lambda_{j}^{*}=\hat{\lambda}_{j}^{*} / a=\lambda_{j}^{*} \sqrt{\zeta_{j}^{*}} / a$.

Given Theorem 1 it is easily concluded that the appropriate relaxed master problem is

$$
\min _{y \in Y} g\left(q^{B O P}\right) \text { s.t. } L_{*}\left(y, \lambda^{\left(k_{2}\right)}\right)=\beta_{k_{2}}-\left(\lambda^{\left(k_{2}\right)}\right)^{T} \sigma \leq 0, \quad k_{2}=1 \ldots K_{2}
$$

where $\beta_{k_{2}}=\sum_{j} \lambda_{j}^{\prime *} \zeta_{j}^{\prime *} / a, \lambda_{j}^{\left(k_{2}\right)}=\lambda_{j}^{\prime *} \sqrt{\zeta_{j}^{\prime *}} / a$, and $a=\sum_{j} \lambda_{j}^{\prime *} \sqrt{\zeta_{j}^{\prime *}}$. Furthermore, the solution to problem (Q3) can be obtained from

$$
\begin{aligned}
& \max _{\lambda^{\prime} \in \Lambda}\left\{\min _{x \in X}\left\{\sum_{j} \lambda_{j}^{\prime}\left(\zeta_{j}-\sqrt{\zeta_{j}} \bar{\sigma}_{j}\right)\right\}\right\} \\
& =\min _{\alpha, x \in X}\{\alpha\} \text { s.t. } \zeta_{j}-\sqrt{\zeta_{j}} \bar{\sigma}-\alpha \leq 0, \quad j=1 \ldots n_{q} \\
& =\min _{\alpha, \tau_{j}>0, x \in X}\{\alpha\} \text { s.t. } \zeta_{j}-\tau_{j} \bar{\sigma}-\alpha \leq 0, \quad\left[\begin{array}{cc}
\zeta_{j} & \tau_{j} \\
\tau_{j} & 1
\end{array}\right]>0, \quad j=1 \ldots n_{q}
\end{aligned}
$$

Clearly this is a convex problem that is readily solved to a global solution.

Example 1c: Application of the convexified GBD algorithm to the mass-spring-damper problem results in Table 2 and Figure 4 (again $\epsilon$ is set to $0.01|\mathrm{LBD}|$ ). Now that the $L_{*}$ 

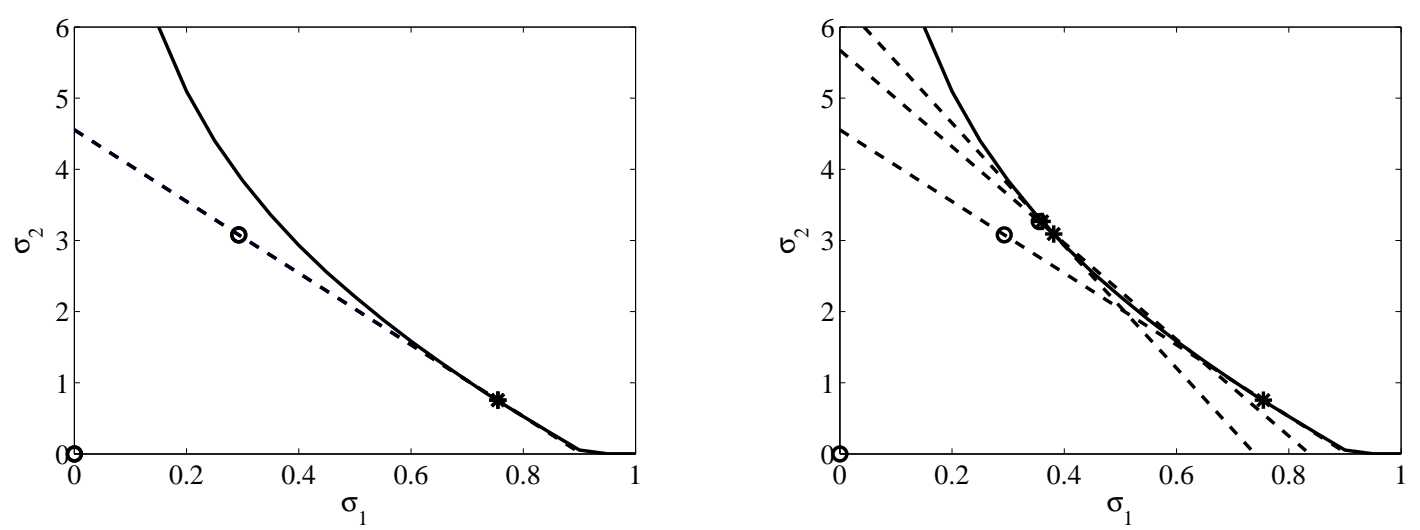

Figure 4: Illustratioin of convexified GBD for Example 1c. See caption of Figure 3 for details.

constraints of RM2 are convex, the problem can be solved significantly faster as indicated in Table 3.

\section{Case Studies}

This section will illustrate the computational scalability of the convexified GBD approach and compare with that of the branch and bound method. In addition, it will be shown that the decomposition allows for the use of a nonlinear steady-state model with virtually no change to the proposed algorithm.

Example 2a: Consider the two CSTR process of Figure 5 (see [3] for the process model details and parameters). The dynamic model of this process contains 6 states, 2 manipulated variables, 5 disturbances and 8 constrained outputs. For the moment assume the steadystate model and objective function are given as linearizations of $g, f$ and $h$ around the OSSOP, as was done in [3]. Using the branch-and-bound approach (which requires the above

Table 2: GBD iteration results for Example 1c

\begin{tabular}{cccccccc}
\hline \hline$k_{2}$ & $\mathrm{LBD}$ & $\mathrm{UBD}$ & $\lambda_{1}^{\left(k_{2}\right)}$ & $\lambda_{2}^{\left(k_{2}\right)}$ & $\left(\zeta_{1}^{*}\right)^{\frac{1}{2}}$ & $\left(\zeta_{2}^{*}\right)^{\frac{1}{2}}$ & $\beta_{k_{2}}$ \\
\hline 1 & -0.707 & -0.245 & 0.834 & 0.166 & 0.755 & 0.755 & 0.755 \\
2 & -0.645 & -0.619 & 0.871 & 0.129 & 0.381 & 3.090 & 0.730 \\
3 & -0.641 & -0.639 & 0.896 & 0.104 & 0.361 & 3.267 & 0.663 \\
\hline
\end{tabular}


Table 3: Comparisons of algorithms for the Mass-Spring-Damper

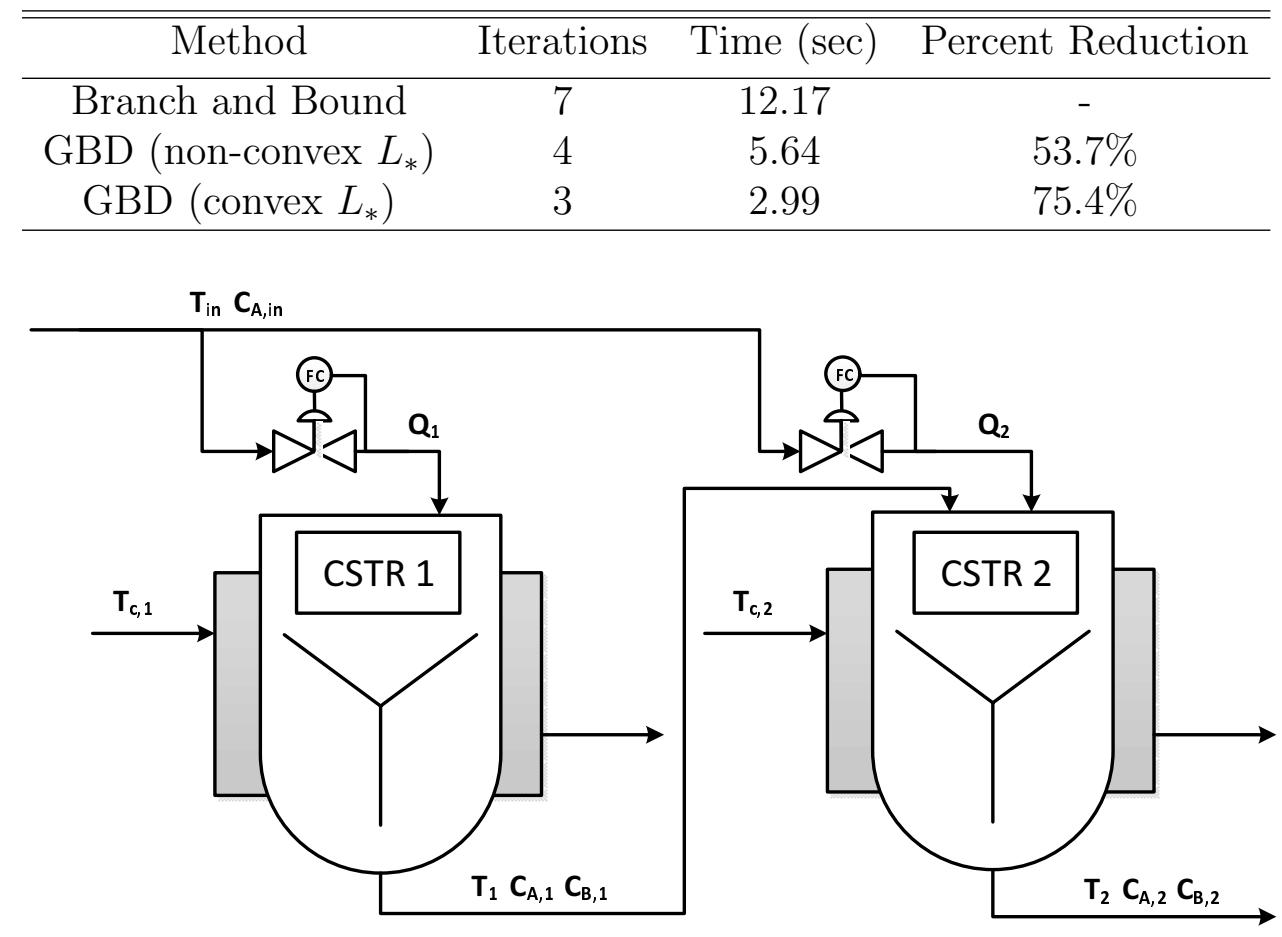

Figure 5: Process diagram for the two-CSTR process

linearizations), the solution required 5879 iterations and a runtime of 46,459 seconds. Using the convexified GBD algorithm, only 12 iterations and 23 seconds were required. Figure 6 illustrates the convergence of the lower and upper bounds, where the UBD was calculated using the method of section 2.1.

Example 2b: Let us now remove the assumption of a linear steady-state model and utilize the nonlinear steady-state model and the nonlinear objective function described in [3]. As suggested previously it would be (nearly) impossible to solve this problem globally using the original branch-and-bound procedure. This is mostly due to a technological issue. Specifically, there currently does not exist an available solver to address general nonlinear optimization problems that also include LMI constraints. However, now that we have decomposed the problem such a solver will not be required. Specifically, Problem Q5 contains only LMI constraints and the relaxed master contains no LMI constraints. Thus, any relevant global algorithm can be used to solve the relaxed master. In the current example, the relaxed 

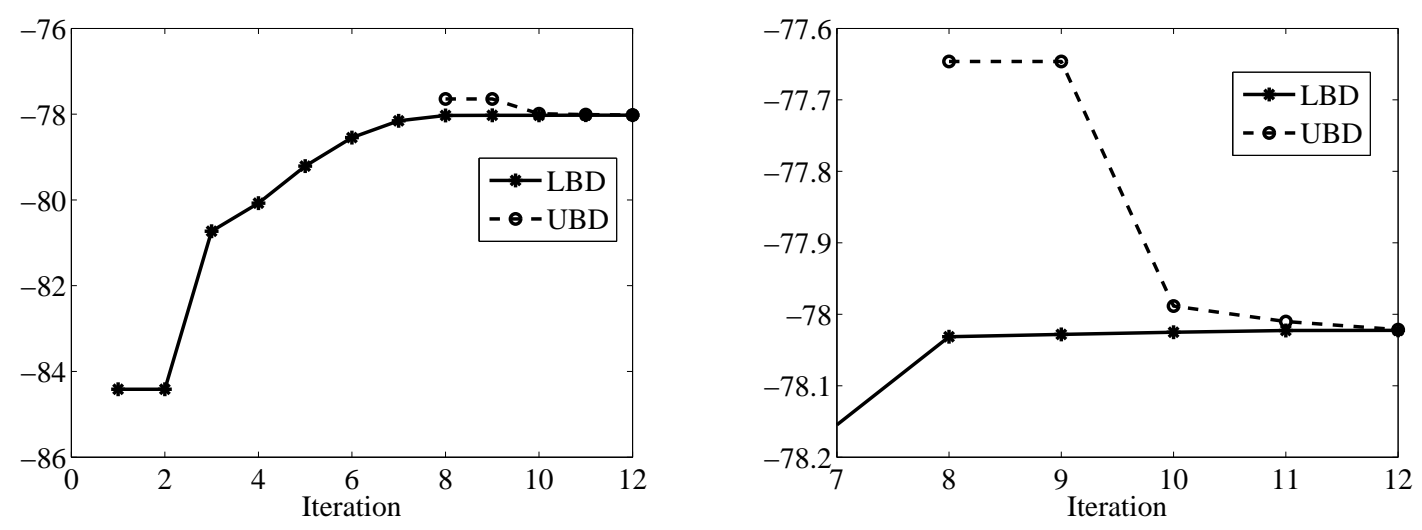

Figure 6: LBD (solid) and UBD (dashed) for Example 2a.

master problem (with nonlinear constraints and nonlinear objective function) is solved using BARON, [25]. In this case, the GBD algorithm required 10 iterations and 30 seconds. A summary of computational effort for the 3 cases of Example 2 is provided in Table 4.

Table 4: Comparisons of computational effort for Example 2

\begin{tabular}{cccc}
\hline \hline Method & Iterations & Time (sec) & Percent Reduction \\
\hline Branch-and-Bound & 5879 & 46,459 & - \\
GBD (Linear steady-state model) & 12 & 23 & $99.95 \%$ \\
GBD (Nonlinear steady-state model) & 10 & 30 & $99.94 \%$ \\
\hline
\end{tabular}

Example 3a: Consider the multi-echelon manufacturing system of Figure 7. The idea is that raw material is ordered and after a delivery delay of $\theta$ weeks it is placed into Storage Unit 0. This raw material can then be processed into product 1 or product 2 and placed into appropriate storage units. To meet consumer demand, products are removed from Storage Units 1 and 2. If the product storage cannot meet demand, then the products must be purchased from a competitor.

In this example the computational impact of $\theta$ will be investigated. The process is inherently discrete-time with a sample period of 1 week. Thus, one will need to solve the discrete-time version of problem (5). This is achieved by replacing (6) with $s^{B O P}=$ 


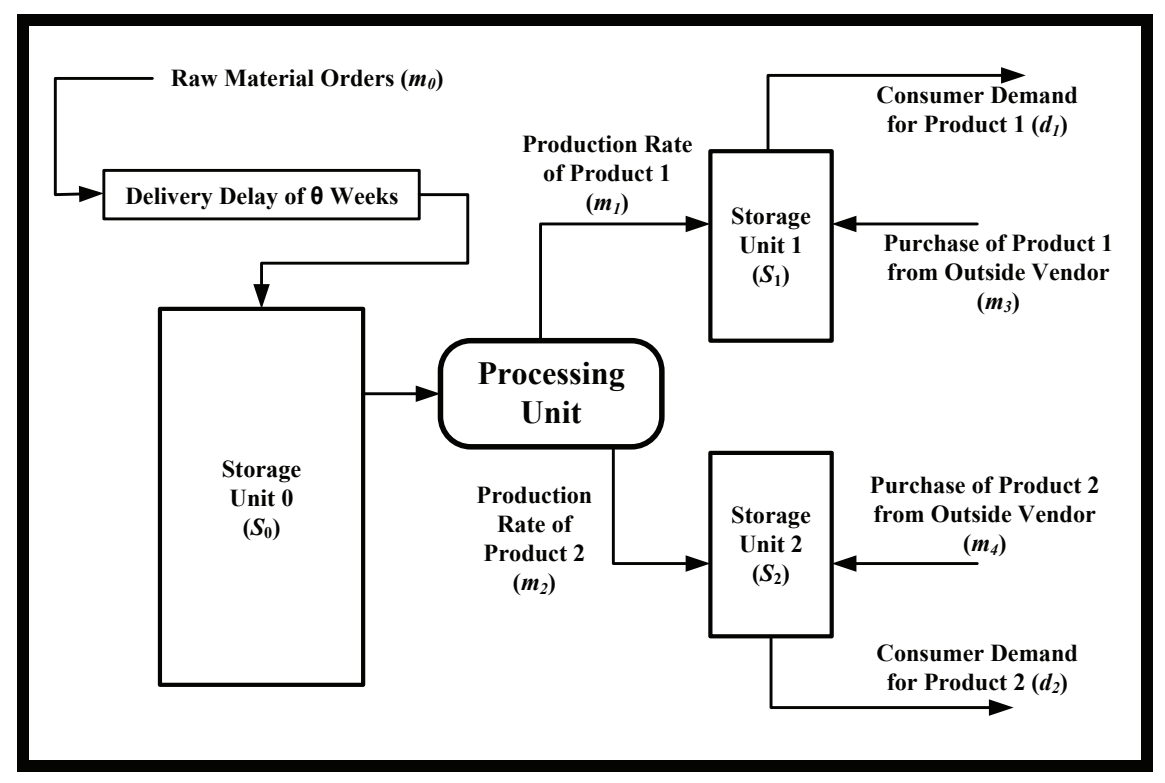

Figure 7: Multi-echelon manufacturing process

$f_{d}\left(s^{B O P}, m^{B O P}, \bar{p}\right) \quad q^{B O P}=h\left(s^{B O P}, m^{B O P}, \bar{p}\right)$ and replacing (9) and (10) with

$$
\begin{aligned}
& {\left[\begin{array}{cc}
M_{0}-G_{d} \Sigma_{p} G_{d}^{T} & \left(A_{d} M_{0}+B_{d} M_{1}\right) \\
\left(A_{d} M_{0}+B_{d} M_{1}\right)^{T} & M_{0}
\end{array}\right]>0} \\
& {\left[\begin{array}{cc}
\zeta_{j}-\rho_{j} D_{p} \Sigma_{p} D_{p}^{T} \rho_{j}^{T} & \rho_{j}\left(D_{x} M_{0}+D_{u} M_{1}\right) \\
\left(D_{x} M_{0}+D_{u} M_{1}\right)^{T} \rho_{j}^{T} & M_{0}
\end{array}\right]>0, j=1 \ldots n_{q}}
\end{aligned}
$$

The manipulated variables are the raw material order, $m_{0}$ (with units of items per week), the production rates of products 1 and $2, m_{1}$ and $m_{2}$, and purchases of products 1 and 2 from outside vendors, $m_{3}$ and $m_{4}$. The disturbances to the process are described by $d_{1, k+1}=$ $0.7165 d_{1, k}+0.2834 p_{1, k}$, and $d_{2, k+1}=0.7165 d_{2, k}+0.2834 p_{2, k}, p_{1, k} \sim N(200, \sqrt{21600})$, $p_{2, k} \sim N(100, \sqrt{9600})$. The processing time of product 2 is twice that of product 1 , which is reflected in the production limitation constraints of the processing unit: $m_{1}+2 m_{2} \leq 450$ items per week. Other constraints on the manipulated variables are: $0 \leq m_{0} \leq 500,0 \leq$ $m_{1} \leq 450,0 \leq m_{2} \leq 225,0 \leq m_{3} \leq 100,0 \leq m_{4} \leq 100$. The three storage units are also constrained: $0 \leq S_{0} \leq 150,0 \leq S_{1} \leq 25$ and $0 \leq S_{2} \leq 25$. In the case of a 3 week delivery 


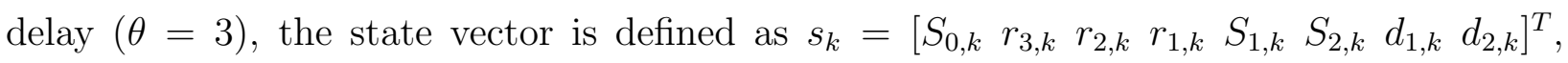
the manipulated variable vector as $m_{k}=\left[m_{0, k} m_{1, k} m_{2, k} m_{3, k} m_{4, k}\right]^{T}$, and the disturbance as $p_{k}=\left[\begin{array}{ll}p_{1, k} & p_{2, k}\end{array}\right]^{T}$. The following process model will result $s_{k+1}=A_{d} s_{k}+B_{d} m_{k}+G_{d} p_{k}$, $q_{k}=D_{x} s_{k}+D_{u} m_{k}$, where $\bar{p}=\left[\begin{array}{ll}200 & 100\end{array}\right]^{T}, \quad \Sigma_{p}=E\left[\left(p_{k}-\bar{p}\right)\left(p_{k}-\bar{p}\right)^{T}\right]=\operatorname{diag}([216009600])$ and $A_{d}, B_{d}, G_{d}, D_{x}, D_{u}$ are given in the appendix.

The economic objective function (to be minimized) is:

$$
\begin{aligned}
g\left(q^{B O P}\right) & =c^{T} q^{B O P} \\
& =50 m_{3}^{B O P}+100 m_{4}^{B O P}+0.5 S_{0}^{B O P}+1.0 S_{1}^{B O P}+1.5 S_{2}^{B O P}
\end{aligned}
$$

where $c=\left[\begin{array}{lllllllll}0.5 & 1.0 & 1.5 & 0 & 0 & 0 & 50 & 100 & 0\end{array}\right]^{T}$. In the general case of a $\theta$ week delivery

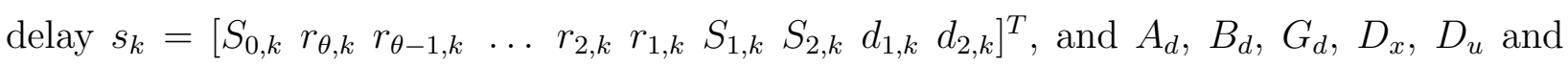
$c$ are appropriately redefined. The solution times for the ELOC problem as a function of $\theta$ are given in Table 5 . It should be noted that larger values of delivery delay, $\theta$, tend to require more safety stock and thus greater utilization of storage facilities. The result is a larger EDOR which will tend to reduce the feasible region of the original problem. For this reason the number of iterations of both algorithms tends to drop for intermediate values of $\theta$. The aspect more relevant to our GBD approach is the fact that the size of the system state is proportional to $\theta$, which will translate into roughly a quadratic growth in number of LMI related variables, $M_{0}$ and $M_{1}$. Thus, the main advantage of the GBD approach, for this example, is that there are significantly fewer calls to the SDP routine. For example in the case of $\theta=15$, GBD makes only 7 SDP calls while the branch and bound makes roughly 206 SDP calls (2 for each iteration). Similarly, for the $\theta=30$ case, the GBD again makes only 7 SDP calls, but each takes about 10 times longer to complete, due to the larger system state. Example 3b: Reconsider Example 3a, but now include the option of augmenting the storage 
Table 5: Comparisons of computational effort for Example 3a.

\begin{tabular}{|c|c|c|c|c|c|}
\hline \multirow{2}{*}{$\begin{array}{c}\text { Delivery Delay } \\
\theta \text { (weeks) }\end{array}$} & \multicolumn{2}{|c|}{ Branch and Bound } & \multicolumn{3}{c|}{ GBD } \\
\cline { 2 - 6 } & Iterations & Time(sec) & Iterations & Time(sec) & Percent Reduction \\
\hline 3 & 122 & 477 & 19 & 23 & $95.2 \%$ \\
4 & 119 & 571 & 16 & 23 & $96.0 \%$ \\
5 & 139 & 712 & 7 & 12 & $98.3 \%$ \\
10 & 120 & 694 & 4 & 10 & $98.6 \%$ \\
15 & 103 & 1122 & 7 & 31 & $97.2 \%$ \\
20 & 100 & 2083 & 7 & 80 & $96.2 \%$ \\
25 & 117 & 4650 & 7 & 155 & $96.7 \%$ \\
30 & 115 & 8369 & 7 & 280 & $96.6 \%$ \\
\hline
\end{tabular}

facilities. This can be done by replacing the storage constraints with the following

$$
\begin{aligned}
& 0 \leq S_{0} \leq 150+\bar{S}_{0} \\
& 0 \leq S_{1} \leq 25+\bar{S}_{1} \\
& 0 \leq S_{2} \leq 25+\bar{S}_{2}
\end{aligned}
$$

where $\bar{S}_{j}$ is the size of the storage unit increase. Then the cost of this augmentation could be added to the objective function

$$
g(q)=c^{T} q+\sum_{j}\left(c_{0, j} \delta_{j}+c_{1, j} \bar{S}_{j}^{0.6}\right)
$$

where $\delta_{j}$ is a $0-1$ integer variable indicating the start-up costs of an augmentation project, which will require the following additional constraints $0 \leq \bar{S}_{j} \leq \delta_{j} \bar{S}_{j}{ }^{\max }, j=0,1,2$ where $\bar{S}_{j}^{\max }$ is a constant indicating the largest possible augmentation. The values of the parameters are assumed to be: $c_{0,0}=2, c_{0,1}=2, c_{0,2}=2, c_{1,0}=1, c_{1,1}=1, c_{1,2}=1$, ${\overline{S_{0}}}^{\max }=50,{\overline{S_{1}}}^{\max }=10$ and ${\overline{S_{2}}}^{\max }=10$.

Clearly, all of these new variables can be added to the set of complicating variables, $y$, and the constraints can be added to $Y$. Thus, the only change will be in the particulars of the relaxed master problem. Given that the relaxed master problem can be solved using any global solver, the fact that the new objective is nonlinear in $\bar{S}_{j}$ and $\delta_{j}$ is an integer variable 
should not be a limitation. For this problem, we again turned to BARON and generated the results of Table 6 . While a branch and bound type solution method is possible, this approach was not investigated as it would have required the development of a customized routine similar to that used in [26].

Table 6: Computational effort for Example 3b

\begin{tabular}{ccc}
\hline \hline Delivery Delay $\theta$ (weeks) & Iterations & Time (sec) \\
\hline 3 & 68 & 395 \\
4 & 69 & 262 \\
5 & 65 & 277 \\
10 & 46 & 278 \\
15 & 50 & 436 \\
20 & 47 & 419 \\
25 & 45 & 1241 \\
30 & 54 & 2757 \\
\hline
\end{tabular}

\section{Conclusion}

In this work we have shown that a novel application of the GBD algorithm to the ELOC problem can yield massive reductions in computational effort. An additional feature of the decomposition is that the use of a nonlinear steady-state process model and a nonlinear objective function, both of which may contain integer variables, is made much easier to implement and is more likely to result in a computationally tractable solution scheme.

A particular aspect of the ELOC problem considered in this work is the fact that noncomplicating variables do not appear in the objective function. As a result the primal problem becomes just a feasibility problem that if found feasible will signal an abrupt stopping of the algorithm. However, in the examples considered the primal problem was never found to be feasible, a characteristic we expect to be true for all ELOC problems of this class. This characteristic of the primal always infeasible also resulted in a lack of UBD calculations, which we circumvented through the development of an ad'hoc UBD estimate. 
In the smart grid coordination version of the ELOC problem [17-19], the objective function is expected to contain non-complicating variables and the primal problem is expected to be feasible at many points within the GBD iteration cycle. Investigation of the GBD algorithm for this class of ELOC problems is the subject of future research.

\section{Acknowledgement}

All authors thank the National Science Foundation (CBET-1511925) for financial support.

\section{References}

[1] Chmielewski DJ, Manthanwar AM. On the tuning of predictive controllers: inverse optimality and the minimum variance covariance constrained control problem. Ind Eng Chem Res 2004;43:7807-7814.

[2] Peng JK, Manthanwar AM, Chmielewski DJ. On the tuning of predictive controllers: the minimum back-off operating point selection problem. Ind Eng Chem Res 2005;44:78147822.

[3] Omell BP, Chmielewski DJ. On the tuning of predictive controllers: impact of disturbances, constraints, and feedback structure. AICHE J 2014; DOI 10.1002/aic.14529.

[4] Bahri PA, Bandoni JA, Barton GW, Romagnoli JA. Back-off calculations in optimizing control: a dynamic approach. Comput Chem Eng 1995;19:S669-S708.

[5] Bahri PA, Bandoni JA, Romagnoli JA. Effect of disturbances in optimizing control: steady-state open-loop backoff problem. Proc System Eng 1996;42(4):983-994. 
[6] Figueroa JL, Bahri PA, Bandoni JA, Romangnoli JA. Economic impact of disturbances and uncertain parameters in chemical processes - a dynamic back-off analysis. Comput Chem Eng 1996;20(4):453-461.

[7] Loeblein C, Perkins JD. Structural design for on-line process optimization: I. dynamic economics of MPC. AICHE J 1999;45(5):1018-1029.

[8] Contreras-Dordelly JL, Marlin TE. Control design for increased profit. Comput Chem Eng 2000;24:267-272.

[9] Zhang Y, Monder D, Forbes JF. Real-time optimization under parametric uncertainty: a probability constrained approach. J Proc Cont 2002;12:373-389.

[10] Soliman M; Swartz CLE, Baker R. A mixed-integer formulation for back-off under constrained predictive control. Comput Chem Eng 2008;32:2409-2419.

[11] Zhao C, Zhao Y, Su H, Huang B. Economic performance assessment of advanced process control with LQG benchmarking. J. Proc Cont 2009;19:557-569.

[12] Ong WK, Durango-Cohen EJ, Chmielewski DJ. Inventory control and LQG: connections and extensions. American Control Conference, Baltimore, MD, 2010.

[13] Omell BP, Chmielewski DJ. Simultaneous BOP Selection and Controller Design for the FCC Process. Proc. of the Am. Cont. Conf., Baltimore, MD, 2010.

[14] Ahmed S, Chmielewski DJ. Power system and controller design for hybrid fuel cell vehicles. American Control Conference, Baltimore, MD, 2010.

[15] Ahmed SK, Chmielewski DJ. On the tuning of predictive controllers for hybrid fuel cell vehicle applications. 10th IFAC International Symposium on Dynamics and Control of Process Systems, Mumbai, 2013.

[16] Omell BP, Chmielewski DJ. On the stability of infinite horizon economic MPC. AICHE Annual Meeting, San Francisco, 2013. 
[17] Omell BP, Chmielewski DJ. IGCC power plant dispatch using infinite-horizon economic model predicitve control. Ind Eng Chem Res 2013;52(9):3151-3164

[18] Mendoza-Serrano DI, Chmielewski DJ. HVAC control using infinite-horizon cconomic MPC. 51st IEEE Conference on Decison and Control, Hawaii, 2012.

[19] Mendoza-Serrano DI, Chmielewski DJ. Smart grid coordination in building HVAC systems: computational efficiency of constrained ELOC. Science and Technology for the Built Environment, accepted 2015.

[20] Van Hessem DH, Scherer CW, Bosgra OH. LMI-based closed-loop economic optimization of stochastic process operation under state and input constraints. 40th IEEE Conference on Decision and Control, Orlanda, Florida, 2001.

[21] Nabil M, Narasimhan S, Skogestad S. Profitable and dynamically feasible operating point selection for constrained processes. J Proc Cont 2014;24:531-541.

[22] Geoffrion AM. Generalized benders decomposition. J Optimiz Theory App 1972;10(4):237-260.

[23] Bagajewicz MJ, Manousiouthakis V. On the generalized benders decomposition. Comput Chem Eng 1991;15(10):691-700.

[24] Floudas CA, Aggarwal A, Ciric AR. Global optimum search for nonconvex NLP and MINLP problems. Comput Chem Eng 1989;13(10):1117-1132.

[25] Tawarmalani M, Sahinidis NV. A polyhedral branch-and-cut approach to global optimization. Math Program 2005;103(2):225-249.

[26] Peng JK, Chmielewski DJ. Optimal sensor network design using the minimally backedoff operating point notion of profit. Proc of the Am Cont Conf, Portland, OR, 2005. 


\section{Appendix}

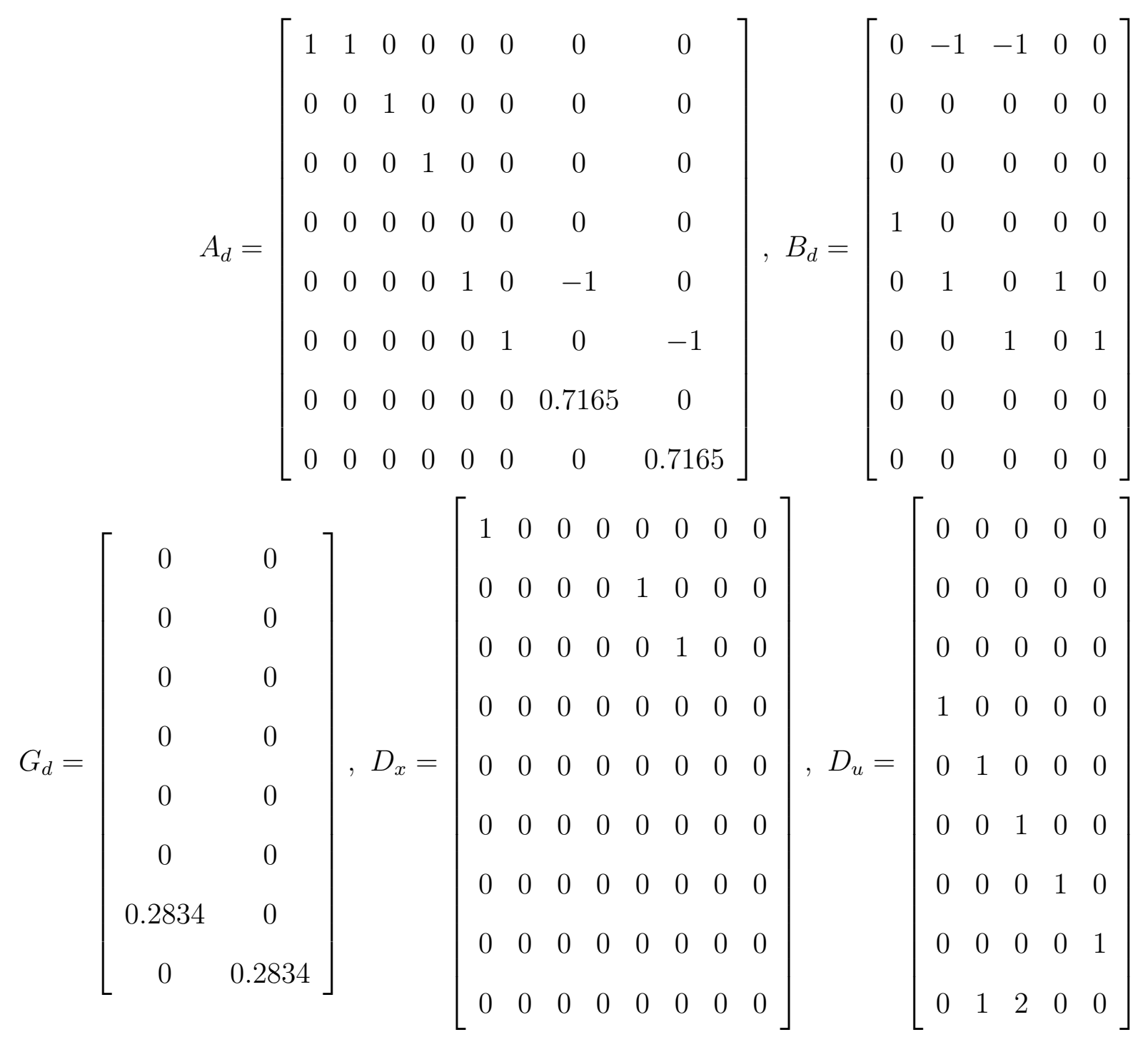

